\title{
Breaking the symmetry: Mirror discrimination for single letters but not for pictures in the Visual Word Form Area
}

\author{
Felipe Pegado $^{\text {a,b,c,* }}$, Kimihiro Nakamura ${ }^{\text {a,b,c,d }}$, Laurent Cohen ${ }^{\text {e,f,g }}$, Stanislas Dehaene ${ }^{\text {a,b,c,d }}$ \\ a INSERM, U992, Cognitive Neuroimaging Unit, F-91191 Gif/Yvette, France \\ b CEA, DSV/I2BM, NeuroSpin Center, F-91191 Gif/Yvette, France \\ c Univ Paris-Sud, Cognitive Neuroimaging Unit, F-91191 Gif/Yvette, France \\ d Collège de France, F-75005 Paris, France \\ e AP-HP, Groupe hospitalier Pitié-Salpêtrière, Department of Neurology, Paris, France \\ ${ }^{\mathrm{f}}$ INSERM, ICM Research Center, UMRS 975, Paris, France \\ g Université Paris 6, Faculté de Médecine Pitié-Salpêtrière, Paris, France
}

\section{A R T I C L E I N F O}

\section{Article history:}

Received 23 June 2010

Revised 23 October 2010

Accepted 12 November 2010

Available online 24 November 2010

\section{Keywords:}

Mirror-images

Reading

VWFA

Priming

Letters

fMRI

\begin{abstract}
A B S T R A C T
Humans and primates can quickly recognize mirror images of previously exposed pictures. This spontaneous mirror invariance, though advantageous for visual recognition, makes it difficult to distinguish the orientation of letters (e.g. to differentiate a "b" from a "d"), and may result in classical mirror reading and writing errors in preschoolers. Mirror invariance must therefore be overcome during reading acquisition. The Visual Word Form Area (VWFA), a region in the ventral stream that develops with reading expertise, was previously shown to discriminate words from their mirror images in literate adults. Here we investigate whether this region underlies mirror-image discrimination at the most elementary level of the orthographic code, the single-letter level. Using an fMRI priming paradigm, we demonstrate that the VWFA distinguishes the left-right orientation of single letters in skilled readers, and yet exhibits mirror invariance for simple pictures of matched complexity. These results clarify how letter shapes, after reading acquisition, escape the process of mirror invariance which is a basic property of the ventral visual shape recognition pathway.
\end{abstract}

(C) 2010 Elsevier Inc. All rights reserved.

\section{Introduction}

Humans, monkeys, pigeons and even octopuses can easily recognize reflected mirror images of pictures previously exposed, although these mirror images induce a very different retinal projection (Sutherland, 1960; Mello, 1965; Hollard and Delius, 1982; Tarr and Pinker, 1989; Logothetis et al., 1995; Rollenhagen and Olson, 2000; Baylis and Driver, 2001; Fiser and Biederman, 2001). This spontaneous "mirror invariance" phenomenon enables one to recognize images which are indifferently seen from a left or right perspective (e.g. left or right profile of a predator) and silhouettes of objects seen from opposite sides, representing an advantage for learning and survival.

In humans this mirror-invariance mechanism has been demonstrated in adults (Tarr and Pinker, 1989; Biederman and Cooper, 1991 ) and is known to be already present in infants (Bornstein et al., 1978). Mirror-invariance poses a challenging problem at the beginning of reading and writing acquisition. Because of the fixed orientation of letters in the Latin alphabet and many other writing

\footnotetext{
* Corresponding author. INSERM U992, Cognitive Neuroimaging Unit, Gif sur Yvette, 91191, France. Fax: +33169087973.

E-mail address: felipepegado@yahoo.com (F. Pegado).
}

systems, and the presence of minimal pairs such as "b" and "d", mirror invariance is an undesirable property for reading. Many children initially make mirror errors in writing and reading, but after a few years of schooling, most become able to tell the correct orientation of letters (e.g. discriminate a "b" from a "d") for reading and writing (Cornell, 1985; Schott, 2007). Dyslexic children who present a specific difficulty in learning to read despite a normal intelligence level can be delayed in the acquisition of correct letter orientation (Terepocki et al., 2002), resulting in paradoxically better performances than controls in a same-different judgement task where mirror letters should be judged as "same" (Lachmann and van Leeuwen, 2007).

The brain's underpinnings of mirror invariance are starting to be uncovered in non-human primates and humans. Monkey's single cell recordings show neurons in visual ventral cortex (IT) responding in an equivalent way to a given picture and its mirror reflected presentation (Logothetis et al., 1995; Rollenhagen and Olson, 2000). In humans, the phenomenon of fMRI repetition suppression has been used to demonstrate that pictures and their mirror images can be encoded as the same object within the ventral stream (Eger, 2004; Vuilleumier et al., 2005; Dehaene et al., 2010). Investigating if written words elicit equivalent mirror priming as pictures in the occipitotemporal cortex is of particular importance since it has been claimed that this region is a domain-general site (Priftis et al., 2003; Price et al. 2006). Indeed, Dehaene et al. (2010) provided fMRI evidence that this 
mirror invariance exists for pictures but not for written words. The same restricted region of the left occipito-temporal cortex, namely the Visual Word Form Area (VWFA), simultaneously showed mirror invariance for pictures and mirror discrimination for words. That is, it no longer showed repetition suppression when a word was preceded by a mirror-image version of itself. This region is known to adapt to orthographic stimuli during reading acquisition across different cultures (Cohen et al., 2000; McCandliss, 2003; Nakamura et al., 2005; Maurer et al., 2008; Dehaene, 2009; Graves et al., 2010), and it was therefore concluded that it may be the site of the loss of mirror invariance which occurs in young children and seems to be needed for efficient reading in alphabetic scripts.

The previous fMRI priming results of Dehaene et al. (2010), however, might simply be taken to mean that participants were unable to extract any significant visual information from the mirrored prime words. Obviously, however, this was the only category of stimuli which could not be identified - perhaps explaining that such stimuli, contrary to normal words, caused no repetition suppression. Furthermore, participants were engaged in a semantic task (object size discrimination), and it could be argued that pictures were nameable and semantically meaningful stimuli in both orientations, while the mirrored words were meaningless and task-irrelevant. Both of these factors, on their own, might explain the absence of mirror priming with mirrored words in the Dehaene et al. (2010) study. The motivation of the present study was therefore to investigate if mirror invariance and its absence for alphabetic stimuli could be replicated at a lower level of the orthographic code, the single-letter level, where these potential artifactual sources could be controlled (Lachmann and van Leeuwen, 2007). Here we presented single letters and complexity-matched pictographic stimuli, as well as their mirrored counterparts, as stimuli in an fMRI repetition suppression study. All stimuli were thus equally simple and relevant to the low-level visual perception task chosen. We investigated whether, in expert readers, the VWFA discriminated letters from their mirror images. We also wondered if other, perhaps earlier visual areas maintained a mirrorinvariance for both letters and pictures.

\section{Material and methods}

Fourteen healthy right handed subjects (mean age 46.5 (20-62), ten females), participated in the present study. All of them were skilled readers of French, and had normal or corrected to normal vision. None had antecedents of neurological or psychiatric diseases. One subject was excluded from the behavioural analysis because reaction time data were missing. All subjects gave written informed consent prior to the fMRI experiment. The present research was approved by the regional ethical committee, as part of a general research program on functional neuroimaging of the human brain which was sponsored by the Atomic Energy Commission (Denis Le Bihan).

The stimuli used for the behavioural task during the fMRI scanner were 12 different single letters strings in Arial font (a, c, e, r, s, z, b, f, g, $\mathrm{h}, \mathrm{k}$, and $\mathrm{y}$ ) and 12 outline iconic pictures matched for size and total number of pixels $(Q, Q, E, \triangle, X, J, \%$, and 1). All stimuli were presented in black on a white băckground and occupied similar locations on screen. Each trial started with a fixation cross and a central frame for $50 \mathrm{~ms}$, then the prime was presented for $50 \mathrm{~ms}$, followed by another fixation cross and frame during $50 \mathrm{~ms}$ and finally the target was presented for $500 \mathrm{~ms}$. Subjects performed a simple feature decision task: they had to judge if the target stimuli stayed within the central frame or whether part of it exceeded this boundary (Fig. 1). They responded by pressing right or left button respectively. Targets were always presented in a fixed orientation, which corresponded to the standard orientation for letters and was selected at random for pictures. Primes could be: 1 / exactly the same as target, 2/ the same but in a mirror oriented presentation, 3/ different in the same orientation or 4 / different in a mirror oriented presentation (see Fig. 1). A $2 \times 2 \times 2$ factorial design was used, with two levels of stimulus category (letters or pictures, presented in distinct fMRI runs), two levels of orientation (normal or mirror) and two levels of repetition (same or different).

All imaging data were collected with a 3 Tesla MRI scanner (Trio 3T, Siemens) in Neurospin Imaging Center at Saclay, France. The following parameters were used: voxel resolution $=3.8 \times 3.8 \times 4 \mathrm{~mm}$, with a gap of $1 \mathrm{~mm}$ between slices; matrix of $64 \times 64,24$ slices per volume, TR $=1.4 \mathrm{~s}$, and angle $=80^{\circ}$. Six runs, each lasting $\sim 7 \mathrm{~min}$ and giving a total of 295 volumes were collected, three for single letters and the other three for iconic pictures, in a counterbalanced order. In each run, the four trial types were presented 40 times in a pseudorandomized order using event-related design. In 40 additional trials within each run, the primes and targets were omitted, thus providing an event-related baseline condition relative to which we could compute activation in the other trials.

A two-level analysis was implemented in SPM5. First, functional images were corrected for head motion, resampled every $3 \mathrm{~mm}$ using tri-linear interpolation, normalized to the standard MNI brain space, and spatially smoothed with an isotropic Gaussian filter ( $5 \mathrm{~mm}$ full width at half maximum). Each individual participant's data was then modelled, within each fMRI run, by eight regressors obtained by convolution of the four experimental conditions with the canonical SPM hemodynamic response function and its time derivative. The model included high-pass filtering (cutoff $128 \mathrm{~s}$ ). For each visual category (pictures and letters), we created four individual contrast maps corresponding to the four combinations of orientation (normal or mirror) $\times$ repetition (same or different), each relative to the baseline. These eight contrast images from the first-level analysis were submitted to a second-level $2 \times 2 \times 2$ factorial ANOVA, with factors of participants and within-subject factors of stimulus category (letters or pictures), repetition (same or different prime) and orientation of the first stimulus (normal or mirror). Unless otherwise stated, we used a voxelwise threshold of $\mathrm{p}<0.001$ uncorrected across the brain volume. Because of our precise hypothesis concerning the lack of mirror invariance for letters in the VWFA, we also investigated this region using a smallvolume search, both within a $6-\mathrm{mm}$ sphere centered on a priori coordinates of the VWFA ( $-42,-57,-15$; Cohen et al., 2002), and within a mask formed by the voxels showing mirror priming in our previous study (Dehaene et al., 2010).

\section{Results}

Subjects performed quite accurately, presenting a mean accuracy during feature decision task of $93.4 \%$ (standard deviation [SD] $=8.9 \%)$ for pictures and $93.3 \%(\mathrm{SD}=10.6 \%)$ for letters. Participants responded to letters as quickly as to pictures $(p>0.1)$ (Fig. 2). The main effects of orientation and repetition priming were both highly significant $(p<0.001$ for both). These effects interacted with each other $(p<0.001)$, as the effect size of priming was greater when the primes and targets were in the same orientation (identity priming"; $70 \mathrm{~ms}$ effect, $\mathrm{p}<0.001)$ than when they were left-right flipped ("mirror priming"; $50 \mathrm{~ms}$ effect, $\mathrm{p}<0.001$ ) (see Fig. 2). These priming effects were overall greater for pictures relative to letters $(p<0.005)$. The triple interaction between the three effects did not reach significance $(\mathrm{p}>0.1)$, and in fact priming was significant in all four conditions, including mirror-priming for letters $(43 \mathrm{~ms}$, $\mathrm{p}<0.001$ ). Thus, at the behavioural level we could not reveal any difference between the two categories concerning the priming effects of mirror pairs.

At the brain level, we first studied activations relative to rest. Both letters and pictures activated a similar bilateral fronto-parietotemporo-occipital network, including strong activations in the bilateral ventral stream, extending from the occipital pole to about 
same, normal

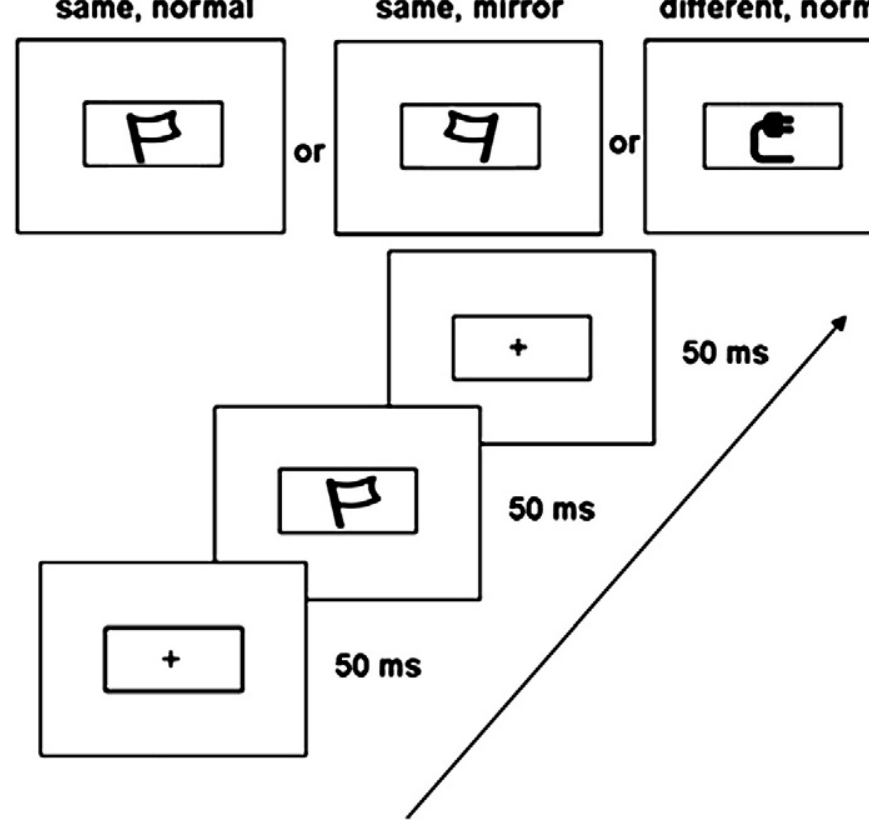

\section{different, mirror}

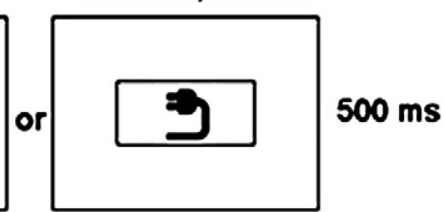

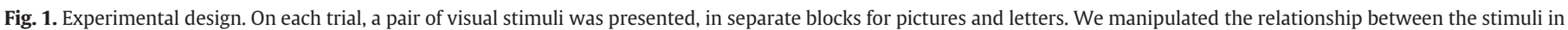

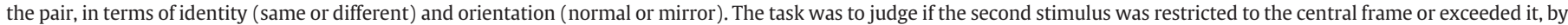
pressing the right or left button respectively. For details see the main text.

MNI $y=-35$. Activations were stronger for pictures than for letters in bilateral occipito-temporal lateral regions, predominantly in the right hemisphere (right: $51,-72,0 ; Z=4.58 ; 82$ voxels; left: $-42-813$; $Z=4.19 ; 29$ voxels). Conversely, letters showed no greater activation relative to pictures at the standard threshold of $\mathrm{p}<0.001$.

In order to examine the main effect of repetition, we contrasted trials in which the prime and the target represented the same item versus trials containing different exemplars, irrespective of their orientation. There was repetition suppression in a large part of the activated bilateral fronto-parieto-temporo-occipital network, including the ventral visual stream. Repetition suppression for pictures was present in the occipito-temporal cortex bilaterally (left: $-39,-81$, $-6 ; Z=5.35,344$ voxels; right: $48,-72,-3 ; Z=5.34 ; 819$ voxels) as well as in parietal $(27,-54,51)$ and motor areas $(-30,-6,60)$. Repetition reduction for letters was found in occipito-temporal cortex bilaterally, but with a slight left-hemisphere predominance (left:

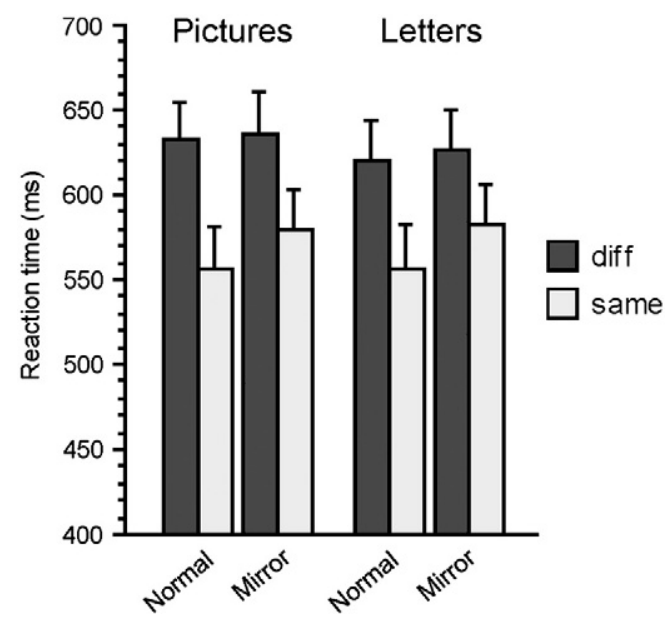

Fig. 2. Behavioural results (reaction times) during the fMRI experiment. Repetition priming effects were found for identity primes and mirror-image primes, irrespective of visual category. This priming pattern did not differ between pictures and letters at the behavioural level, because the triple interaction between repetition, orientation and visual category did not reach significance (see Results).
$-45,-72,-6 ; Z=4.52 ; 105$ voxels; right: $39,-60,-6 ; Z=4.04$; 39 voxels). No significant effect of repetition enhancement was found for pictures. For letters, only the anterior medial frontal cortex showed repetition enhancement effect $(-3,51,3 ; Z=3.97$; 48 voxels).

We then searched for a possible main effect of orientation. When we tested the orientation effect in [normal $>$ mirror] direction, no effect was found. In contrast, when testing the [normal $<$ mirror] contrast we found a bilateral occipito-temporal activation (right: 51 , $-69,0 ; Z=5.42,159$ voxels; left: $-45,-81,3 ; Z=5.22 ; 68$ voxels).

We then studied the interaction of the main factors of category and repetition, looking for voxels showing larger or smaller repetition suppression for pictures relative to letters. First, we started by verifying the effect of repetition suppression (same $<$ different) restricted to pairs presenting the same orientation, i.e. "identity priming" effects. For pictures, we found bilateral occipito-temporal lateral and posterior regions, predominantly in the right hemisphere (right: $51,-69,0 ; Z=6.85 ; 1203$ voxels; left: $-45,-78,-6$; $Z=6.25$; 464 voxels), as well as fronto-parietal activations (see Fig. 3A). Likewise, the equivalent analysis for letters showed a similar ventral visual network bilaterally (left: $-45,-72,3 ; Z=5.37$; 160 voxels; right: $42,-60,-9 ; Z=5.20 ; 189$ voxels).

Our next analysis focused on the critical "mirror priming" effects, i.e., repetition suppression (same<different) for mirror-reversed pairs, which index orientation discrimination. First, when both pictures and letters where collapsed, we found a main effect of mirror priming only on the left occipito-temporal cortex, exactly at the VWFA coordinates $(-42,-60,-9 ; Z=3.91 ; 19$ voxels $)$. In order to understand the contribution of each category to the mirror priming effect, we restricted the analysis to pictures, and again significant repetition suppression was found in the same region $(-42,-57$, $-12 ; Z=3.43 ; 19$ voxels) (see Fig. 3B). Although cluster extent was small and did not reach significance at cluster-size $p<0.05$, corrected for multiple comparisons across the entire brain volume, the effect occurred exactly at the VWFA site expected from our previous mirrorpriming work (Dehaene et al., 2010) (and see later for further analyses with a-priori regions of interest [ROIs]). Additionally, we found small clusters presenting mirror priming for pictures in bilateral premotor cortex (left: $-27,-9,54 ; 15$ voxels; right: 48, 6, 
A

pictures
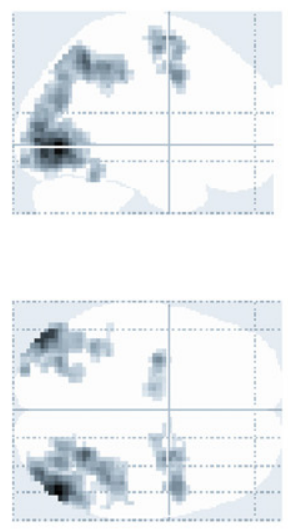

B

pictures
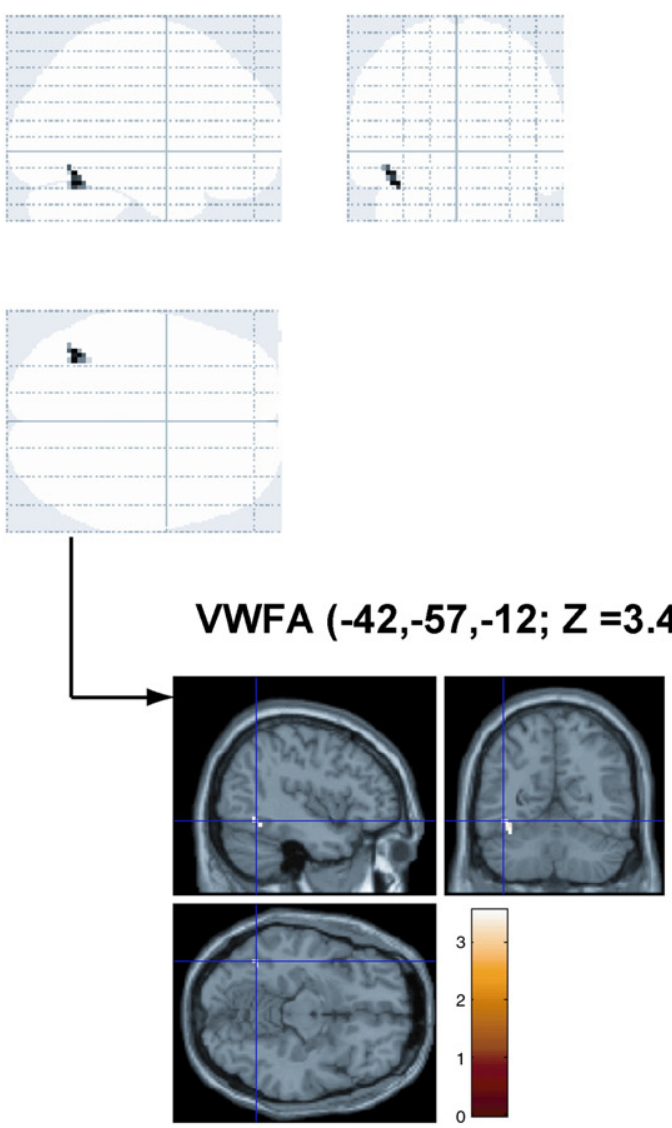

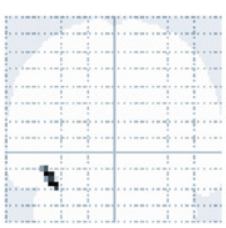

identity priming
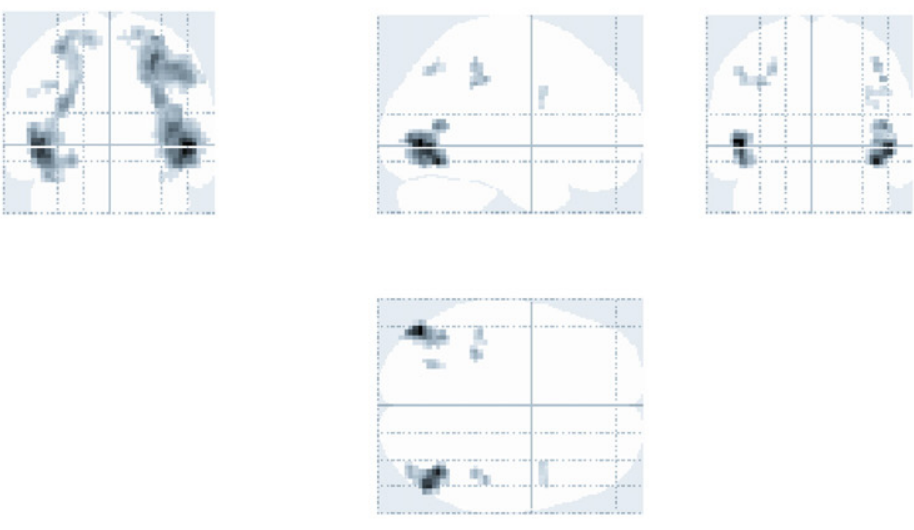

\section{mirror priming}

letters
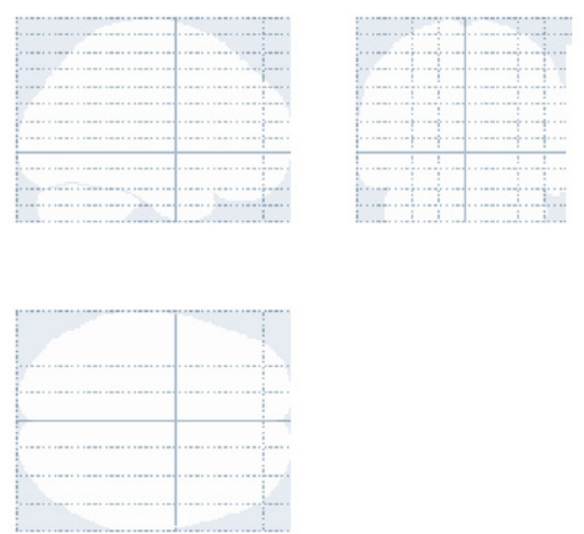

letters

\section{.

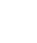


A

Two-way interaction (greater mirror repetition suppression for pictures than for letters)
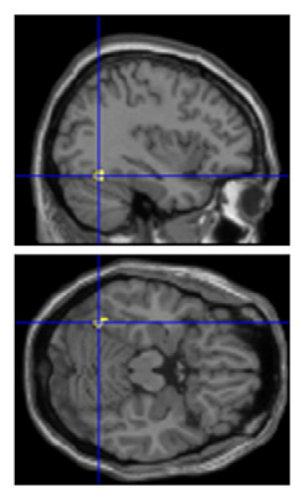
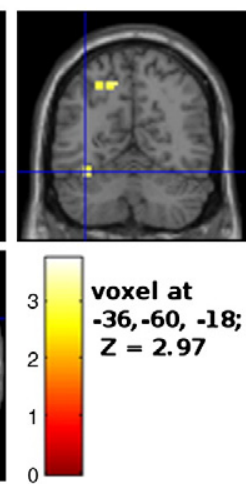

B

Three-way interaction (greater repetition suppression for same pairs than for mirror-oriented pairs, for letters than for pictures)
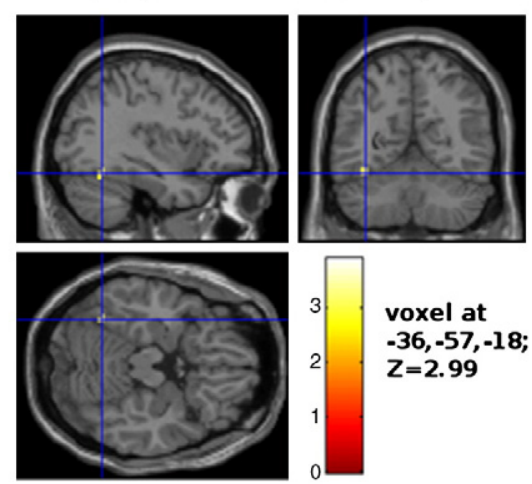

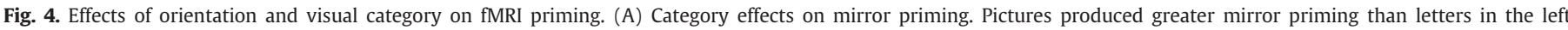

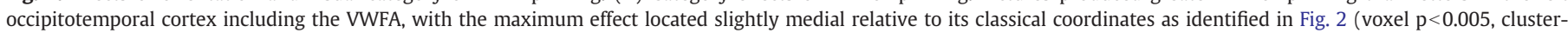

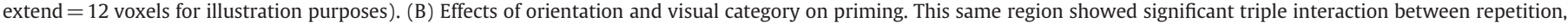
orientation and category (see Results for further analysis) (voxel $\mathrm{p}<0.005$, cluster-extend $=5$ voxels for illustration purposes).

level threshold to $\mathrm{p}<0.005$, pictures showed greater mirror repetition suppression than letters in the left occipito-temporal region $(-36$, $-60,-18 ; Z=2.97 ; 13$ voxels; voxel-level 0.005 ) and left parietal cortex $(-12,-57,-51)$ (see Fig. 4A). Inversely, letters did not show more mirror priming than pictures. These results suggest better orientation discrimination for letters than for pictures at left occipitotemporal cortex, at coordinates previously described as the VWFA.

We probed the significance of these results with two small-volume correction analyses using two independent ROIs arising from previously published studies. Our first ROI was a spherical one and included all voxels within a $6-\mathrm{mm}$ radius of the a priori coordinates of the VWFA (Cohen et al., 2002). The second ROI comprised all voxels in the left occipito-temporal region which showed a significant effect of mirror priming for pictures in our previous study (Dehaene et al., 2010; masking threshold $\mathrm{p}<0.001)$. We then used both ROIs as smaller search volumes for the mirror-priming effect in the present study, separately for each of the two stimulus categories. The first ROI analysis showed a robust mirror priming effect for pictures in the classical VWFA $(-42,-57,-12, \mathrm{Z}=3.43, \mathrm{p}=0.003$, FDR-corrected for multiple comparisons over the ROI). Using the second ROI, we also found a significant effect of mirror priming for pictures at a slightly shifted location $(-36,-57,-18 ; \mathrm{Z}=3.41 ; \mathrm{p}=0.004$, FDR-corrected). In contrast, for letters, neither of these two complementary analyses showed a significant effect of mirror priming for letters.

ROIs were also searched for the 2-way interaction of repetition (different $>$ same) $\times$ category (pictures $>$ letters) for mirror pairs. Both the $6 \mathrm{~mm}$-sphere and the second ROI showed a significant effect (respectively at $-36,-57,-15 ; \mathrm{Z}=2.94 ; \mathrm{p}<0.04$ FDR-corrected and at $-36,-60,-18 ; Z=2.97 ; \mathrm{p}<0.03$ FDR-corrected).

Finally, we looked at the interaction term which compared the magnitude of identity priming (for same-orientation pairs) and of mirror priming (for mirror-oriented pairs). For this analysis we used a slightly lower voxel-level threshold $(\mathrm{p}<0.005)$. For pictures we found identity priming greater than mirror priming in bilateral occipitotemporal posterior regions (right: $45,-69,3 ; Z=5.12 ; 490$ voxels; left: $-42,-81,-3 ; Z=4.42 ; 173$ voxels). For letters, the interaction was found in the left posterior occipito-temporal region $(-45,-78$, $6 ; Z=3.83 ; 23$ voxels), right occipito-temporal cortex $(42,-63,-9$; $Z=3.61 ; 106$ voxels) and left parietal region $(-27,-60,51$; $Z=3.19$; 36 voxels). Crucially, the triple interaction (orientation [ mirror $>$ normal] $\times$ repetition [different $>$ same] $\times$ category [letters $>$ pictures]) showed a significant effect at the VWFA site $(-36$, $-57,-18 ; Z=2.99 ; 6$ voxels) indicating greater mirror priming than identity priming for letters than for pictures in this region (Fig. 4B). Using a small-volume correction with ROIs as previously described, this triple-interaction effect reached significance both when searching around the a priori coordinates of the VWFA ( $\mathrm{p}=0.02$ FDR-corrected) and also within the functional ROI from our previous study $(p=0.03$, FDR-corrected). Thus, this restricted area in left occipito-temporal cortex showed greater mirror image discrimination for letters than for pictures.

For pictures, we further examined possible differences in the magnitude of repetition reduction between pictures with an internal axis of symmetry (e.g. $\boldsymbol{X}$ and $\boldsymbol{K}$ ) and pictures that are intrinsically asymmetric (e.g. $\boldsymbol{\mathcal { X }}$ and $\boldsymbol{\mathcal { K }}$ ). This analysis was motivated by the possibility that mirror-identity could be easier to detect in the former than in the latter, which are more similar to the letters we used. However this additional analysis showed that no brain region showed significant differences between these two subcategories (inclusively masked at $\mathrm{p}<0.05$ by the main effect of repetition suppression for pictures).

In order to compare the magnitude of repetition suppression between single letters and whole words in the VWFA, we performed a joint analysis of the present data and our previous dataset with whole-word stimuli (Dehaene et al., 2010). We constructed a new ANOVA comprising within-subject factors of repetition (same or different prime) and orientation of the first stimulus (normal or mirror) and a between-subject factor of length (letters or words). We found a stronger response $\sim 10 \mathrm{~mm}$ anterior to the classical VWFA coordinates for the triple interaction between repetition $\times$ category (words or letters) $\times$ orientation $(-42,-45,-9 ; \mathrm{Z}=5.89 ; \mathrm{p}<0.001$, FDR-corrected for the brain volume). Therefore, at this site, mirror discrimination was greater for words than for single letters.

\section{Discussion}

In the present study, we provided direct evidence with a priming fMRI paradigm that the VWFA exhibits a sensitivity to the left-right orientation of single letters, i.e., an early and elementary stage of visual word recognition, while this was not the case for nonalphabetic pictures of equivalent complexity (simple iconic images).

At the behavioural level, we observed only a non-significant trend for smaller mirror priming for letters than for pictures, possibly reflecting the fact that behavioural measures can be less sensitive than direct brain-evoked responses (Naccache and Dehaene, 2001). The fMRI results, however, reflected the expertise of good readers to 
detect single letters in a fixed orientation, which does not happen for other kinds of images in general. Our fMRI results support the idea that the VWFA plays a crucial role in the specific discrimination of letter orientation in literate adults. Together with a previous fMRI study from our group (Dehaene et al., 2010) the present work provides further evidence supporting the participation of this restricted region in mirror-orientation discrimination for both letters and words. This finding does not necessarily contradict the "local combination detector" (LCD) model of reading, which assumes a hierarchical coding of letters, bigrams, morphemes and short words within partially distinct sectors of ventral visual cortex (Dehaene et al., 2005), as supported by fMRI activation and priming experiments (Dehaene et al., 2004; Vinckier et al., 2007). First, when reanalyzing together the words and letters experiments, we did find stronger mirror-discrimination for words than for letters in a region just anterior to the VWFA, consistent with the proposed ventral gradient. Second, neurophysiological recordings suggest that multiple stimulus dimensions may be encoded by intermingled or even identical neurons (Freiwald et al., 2009), compatible with the observed overlap of activations in experiments with letters and with words. The possibility that putative neural detectors for letter, bigram and morphemes are partially intermingled within a given subject is compounded by the fact that all our analyses are performed at the group level, thus obliterating any high-resolution information about the precise spatial distribution of cortical responses.

A possible alternative interpretation of the findings is that the left occipito-temporal cortex was modulated in a top-down manner by higher-order language areas, as suggested for instance by Kherif et al. (2010). According to this interpretation, priming in our experiment would be mediated by top-down feedback from a higher-level representation of the name of the prime onto the subsequent target. Letters would be more strongly associated with their names in canonical than in mirror orientation, while pictures would not show such an orientation difference, thus explaining the asymmetry in mirror priming. Although we cannot completely rule out this alternative interpretation, our experiment was designed to minimize the role of top-down effects arising from language areas. First, we opted for a neutral non-linguistic task (visual feature decision), which could be performed equally well with letters, mirror-letters and pictures. Second, we selected a short presentation of the prime $(50 \mathrm{~ms})$ associated with a very short prime-target SOA (100 ms). We did not use masked primes because it is unclear whether they can cause mirror priming (Bar and Biederman, 1998) and because the present design was kept identical to a previous fMRI study on mirrorimage recognition (Dehaene et al., 2010). Still, our tasks and short presentation times should have minimized late-stage attentional, topdown or strategic effect on prime processing (although Kherif et al. (2010) do leave open the possibility that some top-down influence may have remained). Note also that our whole-brain scans do not provide any evidence that mirror-priming arose from a region outside the ventral visual system, as the left occipito-temporal area was the only region showing such priming, and areas typically involved in naming were not observed (see Fig. 3B).

There is evidence that left-right mirror invariance is deeply entrenched in the ventral visual pathway of all primates, as inferior temporal cortex neurons in monkeys show such an invariance even when using abstract geometric forms as stimuli: neurons that respond preferentially to a given picture also respond in an nearly-equivalent manner to their mirror counterpart, but not to other viewpoints (Logothetis et al., 1995; Rollenhagen and Olson, 2000; Baylis and Driver, 2001). Moreover, mirror invariance was also demonstrated in infants (Bornstein et al., 1978) and may therefore constitute a default invariance of the visual system in humans. As a consequence, it is not surprising that young readers confuse left and right orientation of letters. However, after a couple of years of training they become able to read and write in the correct orientation (Cornell, 1985).
Remarkably, children within the normal range of intelligence but presenting specific difficulty in learn to read (dyslexics) often show a delay in the acquisition of the correct orientation of single letters (Terepocki et al., 2002), resulting in a paradoxical better-than-normal performance in same-different tasks when mirror letters should be assign as "same" (Lachmann and van Leeuwen, 2007). Thus, all the evidence converges to the idea that the early stage of the visual system is one of mirror invariance.

When one learns to read, however, letters should be recognized in a fixed orientation, forcing the visual system to discriminate their orientations in an effortful manner. Longcamp et al. (2008) suggest that a movement code for handwriting gestures, particularly involving the bilateral inferior parietal lobule, may help in disambiguating symmetrical letters. Once reading expertise sets in, the recognition of letters in a fixed orientation (mirror discrimination) becomes automatic and effortless. What is the neuronal mechanism behind this automaticity? The present fMRI results, using short visual primes, indicate that the ventral visual pathway, and precisely the left visual word form, in skilled readers, is the primary site for such efficient left-right discrimination abilities, both for letters and for written words (Dehaene et al., 2010). This finding is strongly convergent with another source of data coming from studies of brain-lesioned patients. Indeed, neuropsychological data suggest that, in expert readers, the visual recognition of mirror images involves distinct neural systems for letters and written words than for other non-linguistic visual objects. Some patients, following parieto-occipital lesions, suffer from a specific visual leftright orientation agnosia, i.e., they become unable to distinguish the left-right orientation of objects, even though they are still able to recognize them (Turnbull and McCarthy, 1996; Davidoff and Warrington, 2001; Priftis et al., 2003; Vinckier et al., 2006). Remarkably, mirror discrimination of letters/words is perfectly intact in these patients. Thus, in skilled readers the dorsal stream may not be necessary for mirror discrimination for this special class of linguistic visual objects. One may speculate that at the beginning of learning to read, the dorsal system probably has an important role in disambiguating the orientation of alphabetic symbols, but after substantial exposure to them in a fixed orientation, the dorsal system is no longer necessary to this task, as the ventral system encodes the orientation of letters. Such a dorsal-to-ventral shift underlying the sensitivity to letter orientation would be consistent with the view that, in the initial stage of learning to read, left-right discrimination of letters is primarily guided by motor representations of handwriting acts encoded in parietal and motor/premotor areas (Longcamp et al., 2008). This hypothesis, however, remains to be directly tested.

Another important open question concerns the impact of the acquisition of reading on other cognitive skills. More precisely, does mirror orientation discrimination in the alphabetic domain transfer to other visual categories? The answer seems to be, at least partially, yes. Preschoolers and illiterate adults are reported to be specifically impaired in discriminating the orientation of abstract non-alphabetic shapes such as an equilateral triangle (Kolinsky et al., 1987). More surprisingly, Pederson, Danziger and colleagues provided behavioural evidence of the inability to discriminate mirror orientation of geometric shapes in literate adults of the Tamil script, a writing system where no mirror pairs like "b" and "d" or "p" and "q" are present. In contrast, mirror discrimination skills emerged in literate readers who mastered both Tamil and Latin alphabets (Danziger and Pederson, 1998; Pederson, 2003). Danziger and Pederson suggest that the attentional process necessary to distinguish these frequent mirror letters would be the basis for mirror-discrimination ability in readers of the Latin alphabet.

In support of this notion, a role for attention in left-right discrimination of non-alphabetic shapes has been evoked in behavioural and fMRI studies (Stankiewicz et al., 1998; Eger, 2004; 
Vuilleumier et al., 2005). The general conclusion of such studies seems to be that both view-invariant and view-dependent representations can be evoked when the subjects are attentive to the stimuli. This conclusion is compatible with our observation of both view-specific, same-orientation priming in large-chunks of bilateral posterior occipito-temporal cortex, and view-independent, mirror-orientation priming at the VWFA site. In the reading domain, there is evidence of a priming effect for mirror words in the VWFA (Lin and Ryan, 2007; Ryan and Schnyer, 2007) when effortful mirror reading tasks were used. In our study, we used a very short presentation paradigm in order to minimize strategic influences. Altogether, these results therefore suggest that mirror discrimination for single letters happens in an automatic way, as was the case in our previous study using whole words (Dehaene et al., 2010), while mirror invariance no longer occurs automatically for mirror letters in expert readers unless effortful mirror reading strategies are deployed.

The neural mechanisms of mirror generalization for pictures remain to be elucidated. Some authors argue that the mirror invariance process is grounded in the symmetrical arrangement of connections across the two hemispheres, as suggested first by Orton in beginning of the 20th century and then refined by Corballis and Beale (1976). Inter-hemispheric connections through the corpus callosum, linking symmetrically regions of each hemisphere point by point, would operate in such a way that a "b" in one hemisphere would become a " $\mathrm{d}$ " in the other (Corballis and Beale, 1976; Dehaene, 2009). Others authors (Rollenhagen and Olson, 2000; Davidoff and Warrington, 2001) stress that mirror discrimination, for identification purposes, does not represent any evident advantage in a natural world when the vast majority of living and non-living forms do not change category under mirror symmetry. It is therefore plausible that the orientation information is simply not coded at all, so that separate representations of original and mirror representations do not exist, which seems coherent with single neuron monkey data (Logothetis et al., 1995; Rollenhagen and Olson, 2000). For letters, however, such distinct representations would have been created, for instance based on the necessity to map them onto distinct phonological or gesturebased codes in higher areas.

Finally, a more fundamental question that deserves attention concerns the phylogenetic and ontogenetic origins of this particular region of the left occipito-temporal cortex (Dehaene, 2009). Why is this specific region the site of both alphabetic learning, and maximal mirror invariance for objects? What was the role of the VWFA before the relatively recent cultural invention of reading and writing? What is the function of this structure before learning to read, and how does it behave in mature but illiterate adults? While the answer to these questions remains open, concerning specifically the issue of mirror orientation, our research predicts that in pre-school children as in adult illiterates, the VWFA may present mirror generalization for pictures but also for single letters and perhaps even for written words. This prediction should be assessed in future studies.

\section{Acknowledgments}

This research was funded by INSERM, CEA, Collège de France, University Paris XI and an Agence Nationale de Recherche (ANR) grant to SD and LC. KN was supported by the Sumitomo Foundation. We would like to thank Antoinette Jobert, Ghislaine Dehaene-Lambertz, Denis Le Bihan, Lucie Hertz-Pannier, Andreas Kleinschmidt, Caroline Huron and the LBIOM team of the NeuroSpin Center for their help at various stages of this research.

\section{References}

Bar, M., Biederman, I., 1998. Subliminal visual priming. Psychol. Sci. 9, 464-468.

Baylis, G.C., Driver, J., 2001. Shape-coding in IT cells generalizes over contrast and mirror reversal, but not figure-ground reversal. Nat. Neurosci. 4, 937-942.
Biederman, I., Cooper, E.E., 1991. Evidence for complete translational and reflectional invariance in visual object priming. Perception 20, 585-593.

Bornstein, M.H., Gross, C.G., Wolf, J.Z., 1978. Perceptual similarity of mirror images in infancy. Cognition 6, 89-116.

Cohen, L., Dehaene, S., Naccache, L., Lehéricy, S., Dehaene-Lambertz, G., Hénaff, M.A. Michel, F., 2000. The visual word form area: spatial and temporal characterization of an initial stage of reading in normal subjects and posterior split-brain patients. Brain 123 (Pt 2), 291-307.

Cohen, L., Lehéricy, S., Chochon, F., Lemer, C., Rivaud, S., Dehaene, S., 2002. Languagespecific tuning of visual cortex? Functional properties of the Visual Word Form Area. Brain 125, 1054-1069.

Corballis, M.C., Beale, I., 1976. The Psychology of Left and Right. Erlbaum, New York

Cornell, 1985. Spontaneous mirror-writing in children. Can. J. Exp. Psychol. 39, $174-179$.

Danziger, E., Pederson, E., 1998. Through the looking glass: literacy, writing systems and mirror-image discrimination. Written Lang. Literacy 1, 153-169.

Davidoff, J., Warrington, E.K., 2001. A particular difficulty in discriminating between mirror images. Neuropsychologia 39, 1022-1036.

Dehaene, S., 2009. Reading in the Brain. Penguin Viking.

Dehaene, S., Jobert, A., Naccache, L., Ciuciu, P., Poline, J.B., Le Bihan, D., Cohen, L., 2004 Letter binding and invariant recognition of masked words. Behavioral and neuroimaging evidence. Psychol. Sci. 15, 307-313.

Dehaene, S., Cohen, L., Sigman, M., Vinckier, F., 2005. The neural code for written words: a proposal. Trends Cogn. Sci. 9, 335-341.

Dehaene, S., Nakamura, K., Jobert, A., Kuroki, C., Ogawa, S., Cohen, L., 2010. Why do children make mirror errors in reading? Neural correlates of mirror invariance in the visual word form area. Neuroimage 49, 1837-1848.

Eger, E., 2004. BOLD repetition decreases in object-responsive ventral visual areas depend on spatial attention. J. Neurophysiol. 92, 1241-1247.

Fiser, J., Biederman, I., 2001. Invariance of long-term visual priming to scale, reflection, translation, and hemisphere. Vis. Res. 41, 221-234.

Freiwald, W.A., Tsao, D.Y., Livingstone, M.S., 2009. A face feature space in the macaque temporal lobe. Nat. Neurosci. 12, 1187-1196.

Graves, W.W., Desai, R., Humphries, C., Seidenberg, M.S., Binder, J.R., 2010. Neural systems for reading aloud: a multiparametric approach. Cereb. Cortex 20 $1799-1815$.

Hollard, V., Delius, J., 1982. Rotational invariance in visual pattern recognition by pigeons and humans. Science 218, 804-806.

Kherif, F., Josse, G., Price, C.J., 2010. Automatic top-down processing explains common left occipito-temporal responses to visual words and objects. Cereb Cortex 2010, bhq063v1-bhq063.

Kolinsky, R., Morais, J., Content, A., Cary, L., 1987. Finding parts within figures: a developmental study. Perception 16, 399-407.

Lachmann, T., van Leeuwen, C., 2007. Paradoxical enhancement of letter recognition in developmental dyslexia. Dev. Neuropsychol. 31, 61-77.

Lin, C.Y., Ryan, L., 2007. Repetition priming without identification of the primes: evidence for a component process view of priming. Neuroimage 38, 589-603.

Logothetis, N.K., Pauls, J., Poggio, T., 1995. Shape representation in the inferior temporal cortex of monkeys. Curr. Biol. 5, 552-563.

Longcamp, M., Boucard, C., Gilhodes, J.C., Anton, J.L., Roth, M., Nazarian, B., Velay, J.L., 2008. Learning through hand- or typewriting influences visual recognition of new graphic shapes: behavioral and functional imaging evidence. J. Cogn. Neurosci. 20 802-815.

Maurer, U., Zevin, J.D., McCandliss, B.D., 2008. Left-lateralized N170 effects of visual expertise in reading: evidence from Japanese syllabic and logographic scripts. J. Cogn. Neurosci. 20, 1878-1891.

McCandliss, B., 2003. The visual word form area: expertise for reading in the fusiform gyrus. Trends Cogn. Sci. 7, 293-299.

Mello, N.K., 1965. Interhemispheric reversal of mirror-image oblique lines after monocular training in pigeons. Science 148, 252-254.

Naccache, L., Dehaene, S., 2001. The priming method: imaging unconscious repetition priming reveals an abstract representation of number in the parietal lobes. Cereb. Cortex 11, 966-974.

Nakamura, K., Dehaene, S., Jobert, A., Le Bihan, D., Kouider, S., 2005. Subliminal convergence of Kanji and Kana words: further evidence for functional parcellation of the posterior temporal cortex in visual word perception. J. Cogn. Neurosci. 17, 954-968.

Pederson, E., 2003. Mirror-image discrimination among nonliterate, monoliterate, and biliterate Tamil subjects. Written Lang. Literacy 6, 71-91.

Priftis, K., Rusconi, E., Umilta, C., Zorzi, M., 2003. Pure agnosia for mirror stimuli after right inferior parietal lesion. Brain 126, 908-919.

Rollenhagen, J.E., Olson, C.R., 2000. Mirror-image confusion in single neurons of the macaque inferotemporal cortex. Science 287, 1506-1508.

Ryan, L., Schnyer, D., 2007. Regional specificity of format-specific priming effects in mirror word reading using functional magnetic resonance imaging. Cereb. Cortex 17, 982.

Schott, G.D., 2007. Mirror writing: neurological reflections on an unusual phenomenon. J. Neurol. Neurosurg. Psychiatry 78, 5-13.

Stankiewicz, B.J., Hummel, J., Cooper, E., 1998. The role of attention in priming for leftright reflections of object images: evidence for a dual representation of object shape. J. Exp. Psychol. Hum. Percept. Perform. 24, 732-744.

Sutherland, N., 1960. Theories of shape discrimination in octopus. Nature 186, 840-844.

Tarr, M.J., Pinker, S., 1989. Mental rotation and orientation-dependence in shape recognition. Cogn. Psychol. 21, 233-282.

Terepocki, M., Kruk, R.S., Willows, D.M., 2002. The incidence and nature of letter orientation errors in reading disability. J. Learn. Disabil. 35, 214-233. 
Turnbull, O., McCarthy, R., 1996. Failure to discriminate between mirror-image objects: a case of viewpoint-independent object recognition? Neurocase 2, 63-72.

Vinckier, F., Naccache, L., Papeix, C., Forget, J., Hahn-Barma, V., Dehaene, S., Cohen, L 2006. "What" and "where" in word reading: ventral coding of written words revealed by parietal atrophy. J. Cogn. Neurosci. 18, 1998-2012.
Vinckier, F., Dehaene, S., Jobert, A., Dubus, J., Sigman, M., Cohen, L., 2007. Hierarchical coding of letter strings in the ventral stream: dissecting the inner organization of the visual word-form system. Neuron 55, 143-156.

Vuilleumier, P., Schwartz, S., Duhoux, S., Dolan, R.J., Driver, J., 2005. Selective attention modulates neural substrates of repetition priming and "implicit" visual memory: suppressions and enhancements revealed by fMRI. J. Cogn. Neurosci. 17, 1245-1260. 